\title{
Maximization of energy efficiency in wireless ad hoc and sensor networks with SERENA
}

\author{
Saoucene Mahfoudh and Pascale Minet* \\ INRIA, Rocquencourt, 78153 Le Chesnay Cedex, France
}

\begin{abstract}
In wireless ad hoc and sensor networks, an analysis of the node energy consumption distribution shows that the largest part is due to the time spent in the idle state. This result is at the origin of SERENA, an algorithm to SchEdule RoutEr Nodes Activity. SERENA allows router nodes to sleep, while ensuring end-to-end communication in the wireless network. It is a localized and decentralized algorithm assigning time slots to nodes. Any node stays awake only during its slot and the slots assigned to its neighbors, it sleeps the remaining time. Simulation results show that SERENA enables us to maximize network lifetime while increasing the number of user messages delivered. SERENA is based on a two-hop coloring algorithm, whose complexity in terms of colors and rounds is evaluated. We then quantify the slot reuse. Finally, we show how SERENA improves the node energy consumption distribution and maximizes the energy efficiency of wireless ad hoc and sensor networks. We compare SERENA with classical TDMA and optimized variants such as USAP in wireless ad hoc and sensor networks.
\end{abstract}

Keywords: Node activity scheduling, wireless ad hoc networks, sensor networks, energy efficiency, network lifetime, spatial reuse

\section{Introduction}

With the increasing number of applications in many domains (such as detection of forest fire or seismic event, wild life protection, building and bridge monitoring, emergency rescue, target tracking, exploration mission in hostile environments and home monitoring), wireless ad hoc and sensor networks have a promising future. However, nodes in such networks can have a limited amount of energy. Moreover, this energy can be very expensive, difficult or even impossible to renew. That is why, energy efficient strategies are needed in order to maximize both network lifetime and the amount of data delivered. The originality of this paper consists in identifying the highest energy consumption of a node and proposing a solution that improves network energy efficiency by scheduling nodes activity according to their traffic rate.

This paper is organized as follows. Section 2 is a brief overview of the state of the art related to energy efficiency and more particularly to the scheduling of wireless nodes activity. In Section 3, we first analyze the node energy consumption distribution in wireless ad hoc and sensor networks and identify the main energy costs. Section 4 presents a new algorithm SERENA, SchEdule RoutEr Nodes Activity, whose originality consists in allowing router nodes to sleep. Time slots are assigned to nodes in a decentralized and localized way. A node is awake only during its slots and the slots granted to its neighbors, it sleeps the remaining time. In Section 5, we evaluate the impact of SERENA on the network lifetime and the amount of user data delivered. We compute the slot reuse. We then quantify the ability of SERENA to improve energy efficiency in wireless ad hoc and sensor networks. Finally, we compare SERENA with classical TDMA and optimized variants such as USAP in Section 6 and we conclude in Section 7.

\footnotetext{
${ }^{*}$ Corresponding author. Tel.: +33 1396352 33; Fax: +33 1396355 66; E-mail: pascale.minet@inria.fr.
} 


\section{State of the art}

\subsection{Energy efficiency}

The energy constrained nature of wireless nodes requires the use of energy efficient strategies to maximize network lifetime. We can classify these strategies in four categories:

- Topology control algorithms [6,7], have been proposed to reduce energy consumption and improve network capacity, while maintaining network connectivity. The key idea to topology control is that instead of transmitting using the maximal power, each node adjusts its power transmission. Thus, energy dissipated in transmission is reduced and a new network topology is created. Topology control algorithms can have several goals:

* Reduce energy consumption (power grows at least quadratically with distance),

* Reduce interferences,

* Improve spacial reuse and mitigate the MAC-level medium contention.

- Other strategies reduce the volume of information transferred by:

* aggregating information is frequently used in data gathering applications. It can use clusters, like [8,9] or not, like [10-12];

* increasing the refreshment period of control information (e.g.; neighborhood discovery, topology dissemination, data gathering tree structure) with distance, like [13];

* avoiding to transfer information to uninterested nodes;

* optimizing network flooding by reducing the number of useless transmissions. The techniques based on multipoint relays (MPRs) perform well as shown in [34].

- Energy efficient routing strategies aim at selecting routes that minimize the energy consumed by the end-to-end transmission of a packet, and avoid nodes with low residual energy, like [14-20]. Multipath routing protocols rely on the following observation: using the lowest energy path leads to energy depletion of nodes along this path and in the worst case may lead to network partition. To avoid this problem and improve network lifetime, these protocols use multipath routing instead of minimum energy path. The authors of [16] show that link disjoint path routing is more energy efficient and node disjoint path routing is more reliable. However, their model does not take interferences into account. Performance evaluations in [16,35] show that the relative benefit of maintaining one additional path strongly decreases with the number of paths maintained, whereas the complexity increases a lot. That is why, maintaining two paths is generally considered sufficient.

- Finally, the last category allows nodes to sleep in order to spare energy, provided that the network and application functionnalities are still ensured, like [21-23].

\subsection{Node activity scheduling}

In this paper, we focus on the last category, because for a wireless node the state consuming the least energy is the sleeping state, as illustrated by Table 1 in Section 3. Moreover, we assume that the sleeping state consumes much less energy than the idle state. This assumption is met for instance, by the IEEE 802.11 and IEEE 802.15.4 nodes. Hence, allowing nodes to sleep significantly extends network lifetime. All solutions scheduling node activity determine time intervals during which a node must be awake and those during which it can sleep, knowing that the application and network functions must always be satisfied. 


\subsubsection{IEEE 802.15.4 solutions}

802.15.4 [5], the MAC layer can operate in two modes: beacon-enabled mode in which all nodes can sleep. A superframe is used to indicate to each node when it can send data and when it can sleep. This mode is used in tree and star topologies. The second mode, non-beacon mode, is used in mesh topology. In this mode only non-routing nodes can sleep. Data intended to these nodes must be kept by their parent. In this paper, we propose a solution allowing any node, even a router node, to sleep.

\subsubsection{Computation of connected sets of active nodes}

Some authors propose in [21] to extend network lifetime by dividing the network nodes in disjoint sets, such that each node set meets the network and application functions. These sets are activated successively, and at any time only the nodes of one set are active. All others nodes are in the sleep state. The problem consists in maximizing the number of disjoint sets. It has been shown NP-complete. The solution proposed is centralized. These authors have shown in [23] that network lifetime can be improved by allowing non-disjoint sets.

In [22], a distributed and localized solution is proposed. It consists in selecting a connected dominating set of sensor nodes (i.e. each node is either in this subset or is a neighbor of a node in this subset). Only the nodes of this set are active. All other nodes can change their state to sleep mode.

\subsubsection{Coloring algorithms}

Some solutions take advantage of spatial reuse to determine the time intervals dedicated to node activity. Indeed, during the same time interval two transmitters can transmit simultaneously and successfully if they do not interfere. Spatial reuse can be obtained by means of a coloring algorithm. In wireless ad hoc and sensor networks, interferences are generally assumed to be limited to two hops. Hence, two transmitters at a distance strictly higher than two transmission range can simultaneously transmit. That is why, we are interested in two-hop graph coloring. The goal of two-hop graph coloring is to color all nodes with a minimum number of colors in such a way that two nodes at a distance less than or equal to two hops have not the same color. The algorithm used must be deterministic and distributed (see for instance $[24,25]$ for one-hop coloring). In wireless ad hoc and sensor networks, distributed coloring algorithms based on decision rounds require less messages than those based on the alternation of proposal/decision rounds, such as Distributed Largest First [26]. Indeed, in a round, a node sends a message to its one-hop neighbors, this message contains its color and the colors of its one-hop neighbors for a two-hop coloring algorithm. It receives the messages from its one-hop neighbors and takes a decision if it has the highest priority. This is the principle of SERENA, as presented in Section 4.

\subsubsection{Slot assignment}

Slot assignment in wireless ad hoc and sensor networks consists in assigning slots to wireless nodes in such a way that the same slot is never used by two transmitters at a distance less than or equal to two hops, assuming that radio interferences are limited to two hops. In such a context, slot assignment and two-hop coloring are very close.

In [27], the number of slots allocated to a node depends on the number of colors seen by this node. Probabilistic algorithms exist too, such as [28,29], where a node randomly chooses a slot. It can use this slot, only if no conflict is detected on this slot up to two-hop. All these solutions are inadequate in case of non-uniform traffic distribution, because the number of slots allocated to a node does not depend on its traffic rate.

The first deterministic solution based on slot assignment is TRAMA [30]. It consists in a neighborhood discovery protocol, a schedule exchange protocol and an adaptive election algorithm that selects the 
transmitter and receiver(s) for each time slot. Only nodes having data to send contend for a slot; notice however, that a node does not know which of its 1-hop and 2-hop neighbors have data to send. The node with the highest priority in its two-hop neighborhood wins the right to transmit in the slot considered. Each node declares in advance its next schedule containing the list of winning slots and for each winning slot its receiver(s). The adaptivity of TRAMA comes at a price: its complexity. FLAMA [31] optimizes TRAMA for data gathering applications in sensor networks. The protocol is simplified both in terms of 1) message exchange (the schedule is no longer sent, the receiver is implicitly the parent of the transmitting node) and 2) processing complexity (the priority of a node is the weighted sum of its traffic rate and a pseudo-random function of the node identifier and slot number). The number of slots allocated by FLAMA to a node with a given traffic rate highly depends on node priority computation.

With regard to slot assignment, we focus on a deterministic algorithm, less complex than TRAMA and more generic than FLAMA, ensuring that each node is guaranteed to receive at least one slot, and the number of slots granted to a node depends on its traffic rate. Moreover, late node arrivals can create conflicts. The impact of a late arrival should remain localized. We will compare the performance obtained with this of classical TDMA and its optimizations like USAP [32,33].

\section{Analysis of the node energy consumption distribution}

We focus on the distribution of the node energy consumption in a wireless ad hoc network, in order to highlight the main energy costs and then to propose a strategy for improving the network energy efficiency.

\subsection{Energy model}

A wireless node can take four different states with regard to energy:

- Sleeping: the radio is turned off, and the node is not capable of detecting signals: no communication is possible. The node uses the power $P_{\text {sleep }}$ that is largely smaller than in any other state: the energy consumption is minimum;

- Idle: even when no messages are being transmitted over the medium, the nodes stay idle and keep listening the medium with $P_{\text {idle; }}$;

- Transmitting: node is transmitting a frame with transmission power $P_{\text {transmit }}$;

- Receiving: node is receiving a frame with reception power $P_{\text {receive }}$ This frame can be decoded by this node or not, it can be intended to this node or not.

In Table 1, we report the reference values of $P_{\text {transmit }}, P_{\text {receive }}, P_{\text {idle }}$ and $P_{\text {sleep }}$ taken from a Lucent silver wavelan PC card [1] implementing the IEEE 802.11b protocol. These values are used in the performance evaluation reported in this paper.

Table 1

\begin{tabular}{ll}
\multicolumn{2}{c}{ Power value in each radio state } \\
\hline State & Power value \\
\hline Transmitting & $P_{\text {transmit }}=1.3 \mathrm{~W}$ \\
Receiving & $P_{\text {receive }}=0.9 \mathrm{~W}$ \\
Idle & $P_{\text {idle }}=0.74 \mathrm{~W}$ \\
Sleeping & $P_{\text {sleep }}=0.047 \mathrm{~W}$ \\
\hline
\end{tabular}




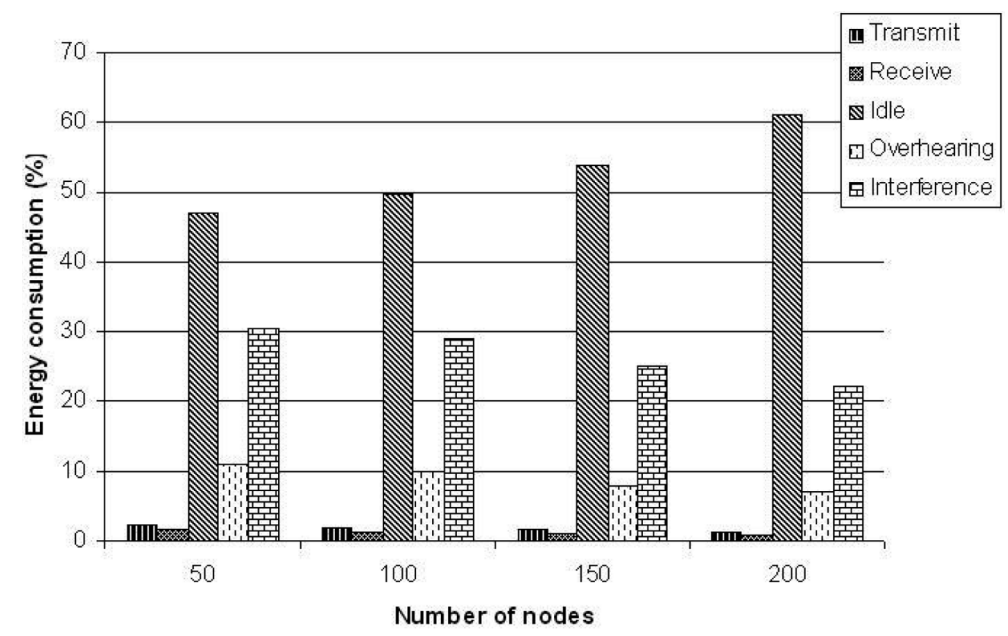

Fig. 1. Distribution of node energy consumption without sleeping state.

When a transmitter transmits one packet to next hop, because of the shared nature of wireless medium, all neighbors of the source receive this packet even if it is intended to only one of them. Moreover, each node situated between transmitter range and interference range receives this packet but it cannot decode it. These two problems generate loss of energy. So to compute the energy dissipated by one transmission, we must take into account these losses on all the non-sleeping nodes belonging to the interference area of the transmitter [3]. The energy dissipated on the transmitter is equal to $E_{\text {transmit }}=P_{\text {transmit }} *$ Duration. On a non-sleeping node belonging to the interference area of the transmitter, it is equal to $E_{\text {receive }}=P_{\text {receive }} *$ Duration, where Duration is the transmission duration of the packet considered.

\subsection{Energy consumption distribution}

\subsubsection{Simulation parameters}

Simulations have been performed for different wireless networks, where the network density (the average number of neighbors per node) is fixed to 10. Nodes whose number varies from 50 to 200, are randomly distributed in the network area. Network throughput is set to $2 \mathrm{Mbps}$. The initial energy of nodes is equal to 100 Joules. User traffic consists of 30 flows, with randomly chosen sources and destinations, and a throughput of 16 Kbps. The size of a message is 512 bytes. The OLSR [2] routing protocol that provides the shortest route is chosen. Messages of the routing protocol are not taken into account. Each result is the average of 5 simulation runs.

\subsubsection{Simulation results}

Figure 1 illustrates the average on all network nodes of the energy dissipated in the different states defined previously. Notice that the Receiving state has been splitted into three substates:

- Receive: when this node is the message destination,

- Overhearing: when this node is a one-hop neighbor of the transmitter but not the message destination,

- Interference: when this node is a two-hop neighbor of the transmitter.

Furthermore, it clearly appears that in the simulations conditions, the highest part of energy (about $50 \%$ in a network of 100 nodes) is surprisingly dissipated in the idle state. The second highest energy 
cost is due to interferences (about 30\%). In [4], we obtain the same conclusion when the EOLSR energy efficient routing protocol is used instead of OLSR, even if EOLSR actively contributes to energy saving. The third cost is due to overhearing (about 10\%). Finally, the energy dissipated in the Transmit and Receive states are small (about 3\%). From these results, we can conclude that the most efficient strategy consists in allowing nodes to sleep. Solutions already exist for non router nodes: see for instance ZigBee [5]. Our purpose is to propose a new solution allowing router nodes to sleep and to evaluate its benefit on the node energy consumption distribution.

\section{Presentation of SERENA}

\subsection{Principles of SERENA}

SERENA allows a router node to stay awake only during its slots and those assigned to its one-hop neighbors and to sleep the remaining time. The number of slots assigned to a router node is proportional to its traffic rate. In any case, any router node is ensured to have at least one slot per frame period. SERENA is decentralized and localized. It adapts to traffic and topology changes. In SERENA, any node $N$ has a priority equal to the cardinality of the set of nodes up to two-hop, denoted $\mathcal{N}^{2}(N)$. In case of equal cardinalities, the node with the smallest identifier wins. SERENA consists of a two-hop coloring algorithm and a slot assignment algorithm.

The aim of the two-hop graph coloring is to color all nodes with the smallest number of colors, in such a way that two different nodes at a distance less than or equal to two hops do not have the same color. A node $N$ can select its color if and only if all nodes in $\mathcal{N}^{2}(N)$ with a higher priority have already selected their color. It then selects the smallest color unused in $\mathcal{N}^{2}(N)$.

As soon as all nodes in $\mathcal{N}^{2}(N)$ are colored, the slot assignment algorithm starts. Its purpose is to assign time slots to each node. Any node $N$ receives a guaranteed slot which corresponds to its color. Then, it computes $k^{\prime}$ additional slots which depends on its traffic and the visible colors in its neighbors, up to two hops according to the following formula:

$$
k^{\prime}=\left\lfloor\frac{\operatorname{traffic}(N)}{\sum_{i \in \operatorname{VisibleColor}(N)} \operatorname{traffic}(i)} *(\text { Size }-|\operatorname{VisibleColor}(N)|)\right\rfloor .
$$

where:

- Size is the size of the frame;

- $|\operatorname{Visible} \operatorname{Color}(N)|$ denotes the cardinal of the set of colors visible by $N$ up to two-hop;

- $\operatorname{traffic}(N)$ is the bandwidth request of node $N$; it is computed from the traffic submitted by $N$ on the last period and the traffic pending on $N$;

- traffic $(i)$ denotes the highest bandwidth request of nodes having color $i$ up to two hops from $N$. Notice that several nodes in $\mathcal{N}^{2}(N)$ can have the same color: this is perfectly acceptable as long as these nodes are not at a distance less than or equal to two hops. That is why the highest of the bandwidth request must be used in the computation.

Because of the distributed slot assignment algorithm, some of these $k^{\prime}$ slots are not guaranteed. Figure 2 illustrates an example of situation where a node cannot allocate its $k^{\prime}$ slots. 


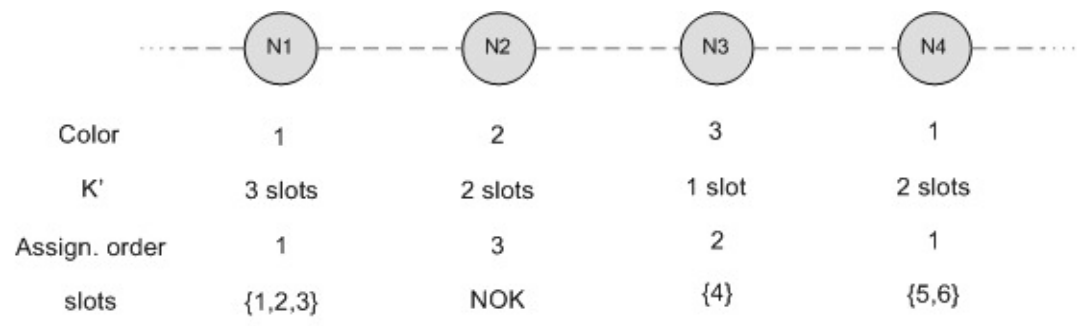

Fig. 2. Distributed slot assignment.

In a distributed and localized slot assignment algorithm, each node locally assigns its slots. We can then face the following situation, where two nodes with a high priority (nodes $N_{1}$ and $N_{4}$ in the example), at a distance $\geqslant 3$ hops reuse the same color and the union of the slots selected by these nodes is not equal to the slots selected by one of them (i.e. $\{1,2,3,5,6\}$ is neither equal to $\{1,2,3\}$ nor to $\{5,6\}$ ). It becomes then impossible for $N_{2}$, at a distance less than or equal to two hops from them, to obtain its two slots: there are no more available slots.

To avoid this problem, we define $k<k^{\prime}$ as the number of guaranteed slots. Hence, $k^{\prime}-k$ slots are requisitionable, where:

$$
k=\left\lfloor\frac{\operatorname{traffic}(N)}{\sum_{M \in \mathcal{N}^{2}(N)} \operatorname{traffic}(M)} *(\text { Size }-|\operatorname{VisibleColor}(N)|)\right\rfloor .
$$

The distinction between $k$ and $k^{\prime}$ comes from the fact that each node is ensured to obtain its $k$ slots, but this is not true with $k^{\prime}$ because of the distributed slot assignment, as illustrated on Fig. 2.

SERENA slot assignment algorithm proceeds as follows: A node $N$ selects its $k^{\prime}$ additional slots among the available ones if and only if all nodes in $\mathcal{N}^{2}(N)$ with a higher priority have already selected their additional slots. If all nodes succeed in assigning their additional slots, the algorithm is over. Otherwise, the node $N$ that is unable to get at least its $k$ additional slots, requisitions them among the requisitionable slots used in $\mathcal{N}^{2}(N)$.

Some optimizations of SERENA are possible, for example ordering the messages to transmit in a slot, in such a way that a node can detect the soonest possible that no message is destinated to it. For instance, we can put the broadcast messages at the beginning of a slot, and order the point-to-point messages by increasing destination identifier. Moreover, a node turns to sleeping mode as soon as it detects that it has no more message to transmit in its slot. Then all its one hop neighbors turns to sleeping mode if they notice, after a timeout, that there are no more messages on the medium.

\subsection{Messages exchanged in SERENA}

SERENA slot assignment algorithm is run periodically to adapt to traffic changes and node mobility. The SERENA message sent by a node $N$ to its one-hop neighbors: contains the following fields:

- the node identifier,

- the node priority,

- the node color,

- the node traffic indication, 
- the list of allocated slots and the indication whether they are requisitionable or not,

- and for each of its one-hop neighbors:

$*$ the node identifier,

$*$ the node priority,

* the node color,

* the node traffic indication,

* the list of allocated slots and the indication whether they are requisitionable or not.

A message loss occurring while SERENA is running, is recovered at the next round. It only induces an additional delay but does not compromise the safety of the algorithm.

\subsection{SERENA and node mobility}

Late node arrivals or node mobility can create color conflicts: two nodes at a distance less than or equal to two hops have the same color. Late node arrivals and node mobility are detected by topology changes. Notice however that a new joining node has no color assigned, whereas a moving node has generally one. Two types of conflict can be created:

- first conflict type: the moving node becomes the one-hop neighbor or two-hop neighbor of a node with the same color. Clearly, this conflict type is possible only in case of mobility.

- second conflict type: two nodes that are at a distance between one and two transmission ranges become two-hop neighbors due to the late arrival of a new node or node mobility.

In the general case, the algorithm proceeds in three steps to deal with late node arrival or node mobility:

- step 1: Conflicts are first detected. The detection is based on the exchange of the colors granted to the node and its one-hop neighbors. If a node $N$ detects that one of the node in $\mathcal{N}^{2}(N)$ has the same color as itself, a conflict exists.

- step 2: Detected conflicts are solved: the node with the highest priority in the conflict keeps its color, the other conflicting nodes get a new color according to SERENA coloring algorithm (i.e. the smallest available color in its neighborhood up to two-hop). In this step, the new node in case of late arrival will also receive its color.

- step 3: This will cause each node seeing a change in colors and bandwidth requests to reassign the slots according to SERENA slot assignment algorithm.

It follows that the impact of a node mobility or a new joining node $N$ is limited to $\mathcal{N}^{2}(N)$.

\section{Performance evaluation}

We now evaluate the performance of SERENA in various wireless ad hoc and sensor networks.

\subsection{Evaluation criteria and simulation parameters}

We consider different wireless ad hoc and sensor networks, characterized by their number of nodes and density. We now evaluate the following criteria:

- SERENA coloring algorithm performance, expressed in terms of number of colors used to color the network, 
- SERENA coloring algorithm complexity, expressed as a number of rounds needed to color the network. By definition, a round allows any node to send a message to its one-hop neighbors, to receive a message from each of them and to process these messages,

- slot reuse is determined by the number of nodes transmitting in the slot considered,

- network lifetime is defined as the time at which a flow destination becomes unreachable.

- user data delivered is the total amount of user data received by their destination in the network,

- distribution of node energy consumption,

- node throughput is the throughput obtained by each node with the slot assignment considered.

The simulation parameters are those given in Section 3.2.1, unless explicitly said.

\subsection{Number of colors and rounds}

We first consider different wireless ad hoc and sensor networks with a density of 10 (the average number of neighbors per node). The number of nodes ranges from 50 to 400 . Figures 3 and 4 represent the numbers of colors and rounds respectively. We observe in Fig. 3 that the number of colors depends very weakly on the number of nodes: it goes from 16.2 to 23.4 , as the number of nodes increases from 50 to 400 . The number of rounds increases more strongly with the number of nodes, as shown on Fig. 4. It reaches 105.8 rounds for a network of 200 nodes.

We now consider a network of 200 nodes where the density varies from 5 to 40 .

The number of colors increases almost linearly with the density, as shown in Fig. 5. Indeed, SERENA coloring algorithm prevents two nodes at a distance less than or equal to two hops to have the same color. Hence, the number of colors depends on the number of nodes up to two hops and then increases with density. Moreover, the number of rounds needed to color all nodes in the network increases with network density, as shown on Fig. 6.

As a conclusion, the number of colors depends strongly on network density and very weakly on node number. Furthermore, the number of rounds depends both on network density and node number.

\subsection{Slot reuse}

We can notice that if all nodes have the same traffic, the average slot utilization is equal to:

$$
\text { average slot use }=\frac{\text { number of nodes }}{\text { number of colors }} \text {. }
$$

We now focus on the slot assignment algorithm and evaluate its performance by means of simulation. We consider a network of 50 nodes. The size of the node queue is set to 50. The other simulation parameters are unchanged. Traffic is not uniformly distributed over the nodes. We evaluate the number of slots obtained by a node with regard to its traffic rate. The slot size is fixed to $12 \mathrm{~ms}$ and the frame consists of 80 slots. The frame size is equal to $2 * 4 *$ density, where $4 *$ density is the average number of nodes up to two hops.

Figure 7 depicts the number of slots assigned to ten nodes. We notice that the nodes with the highest traffic rates (see nodes 3 and 6) receive the highest number of slots (7 and 11 slots respectively), whereas nodes with a small traffic rate (see nodes 1 and 9) receive few slots ( 3 slots for both of them). Simulation results show that as expected, SERENA assigns a slot number proportional to the traffic rate of the node.

Figure 8 depicts the slot reuse. It provides the number of slots shared by 5 nodes, down to 0 node. Two slots among the 80 are empty and 33 slots are reused by three nodes. Three quarter of slots are reused by several nodes. A slot is in average used by 2.43 nodes. This spatial reuse is obtained thanks to the SERENA two-hop coloring and slot assignment algorithms. Hence SERENA results in better network efficiency. 
42 S. Mahfoudh and P. Minet / Maximization of energy efficiency in wireless ad hoc and sensor networks with SERENA

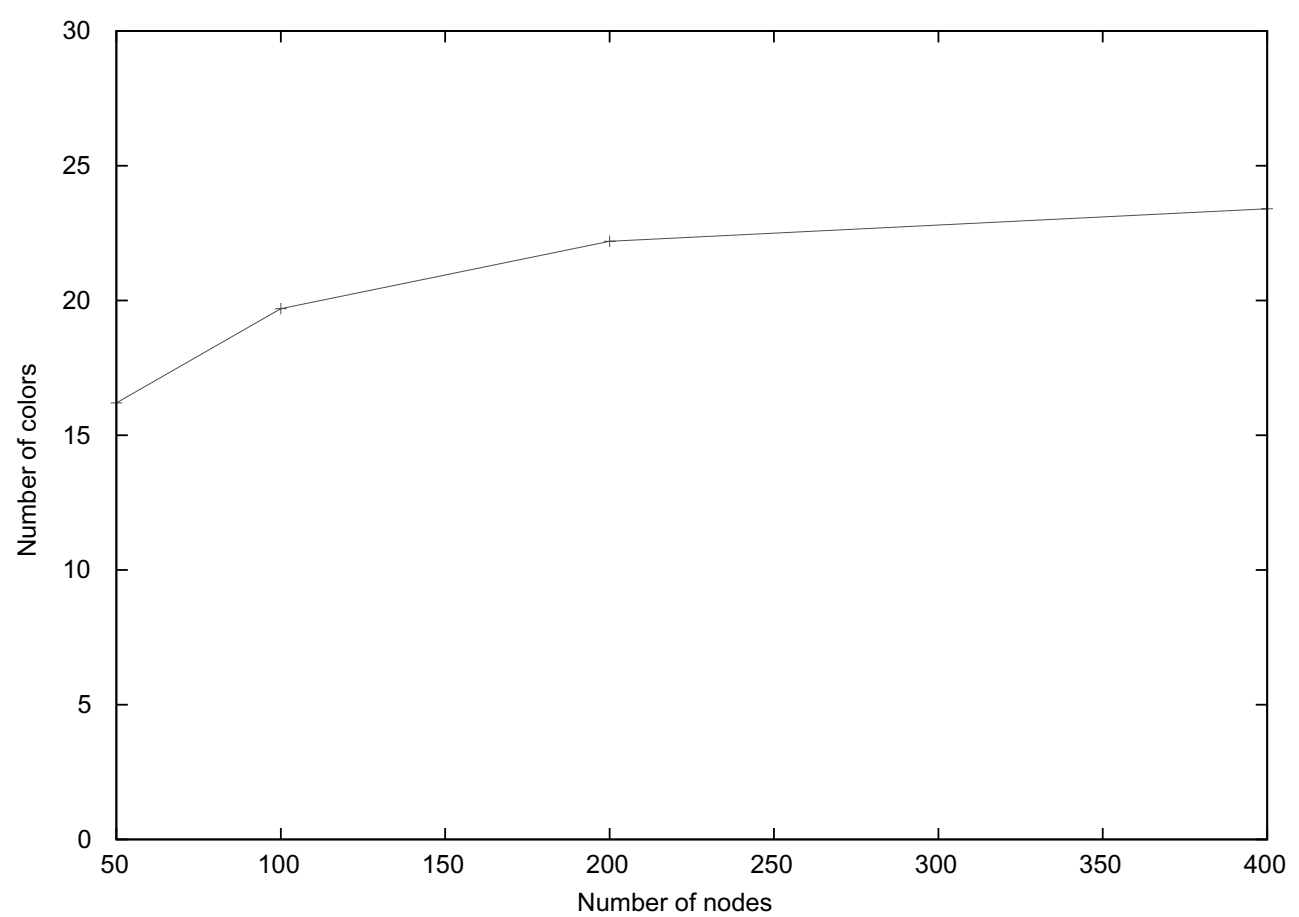

Fig. 3. Number of colors as a function of node number.

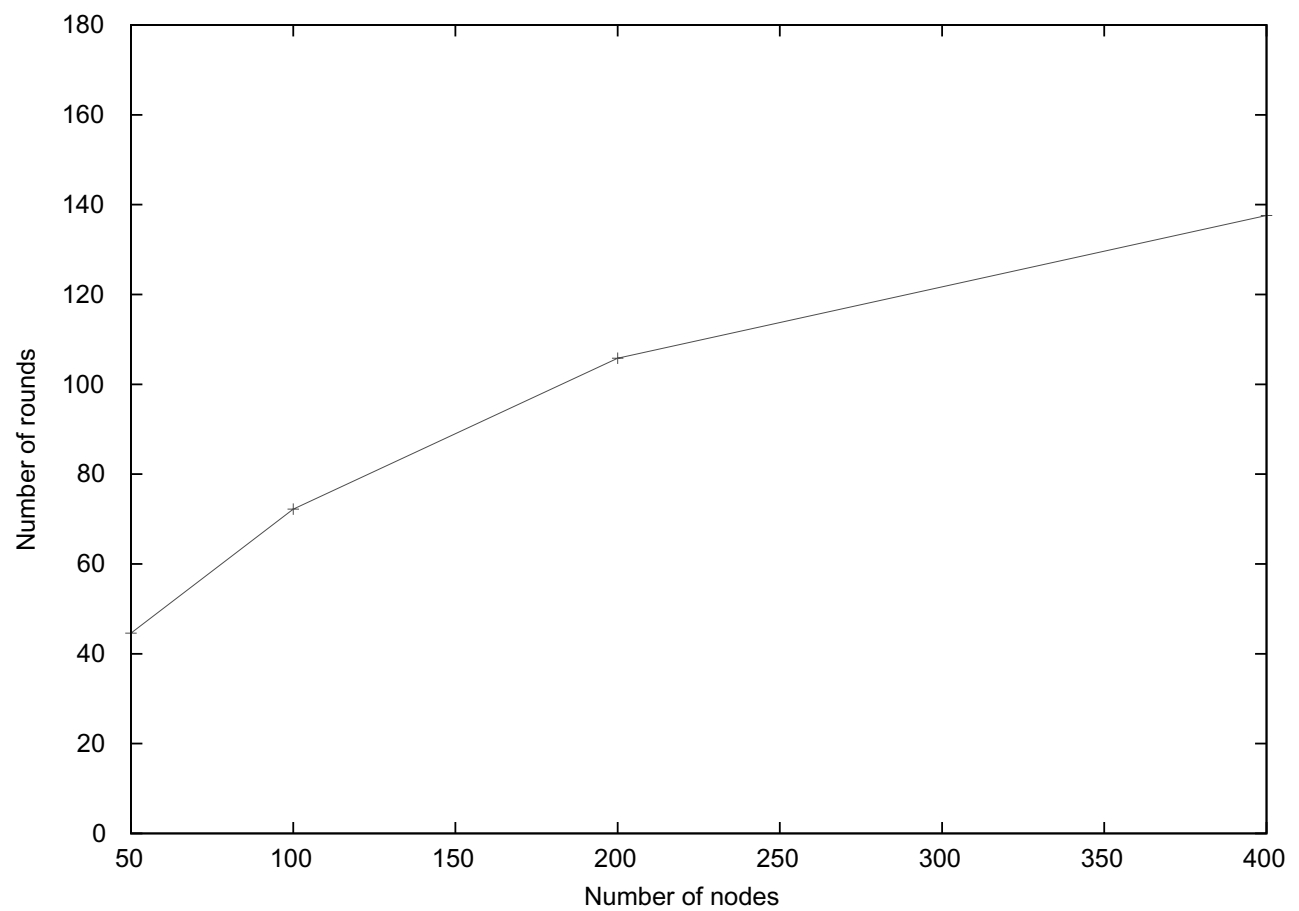

Fig. 4. Number of rounds as a function of node number. 


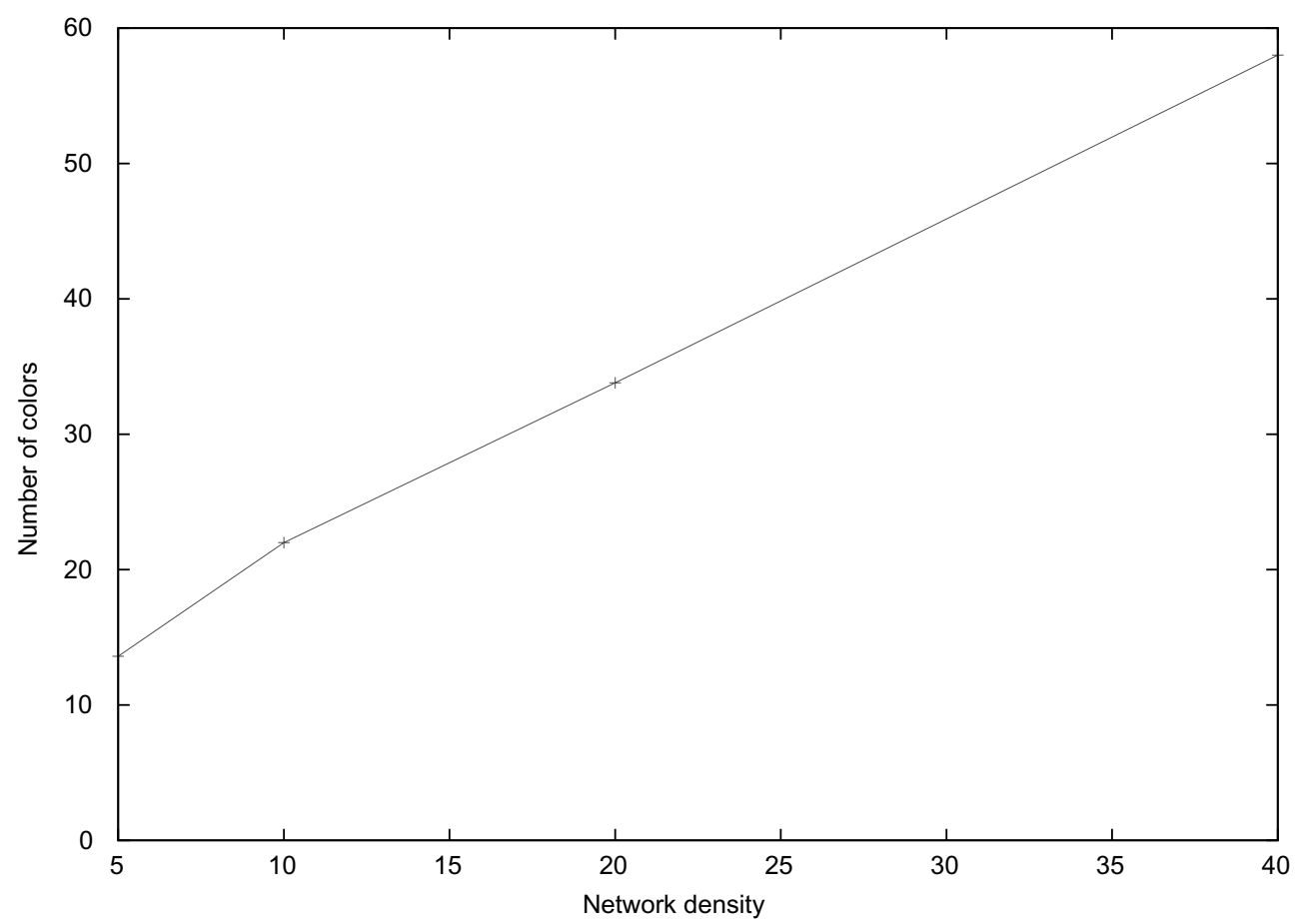

Fig. 5. Number of colors as a function of network density.

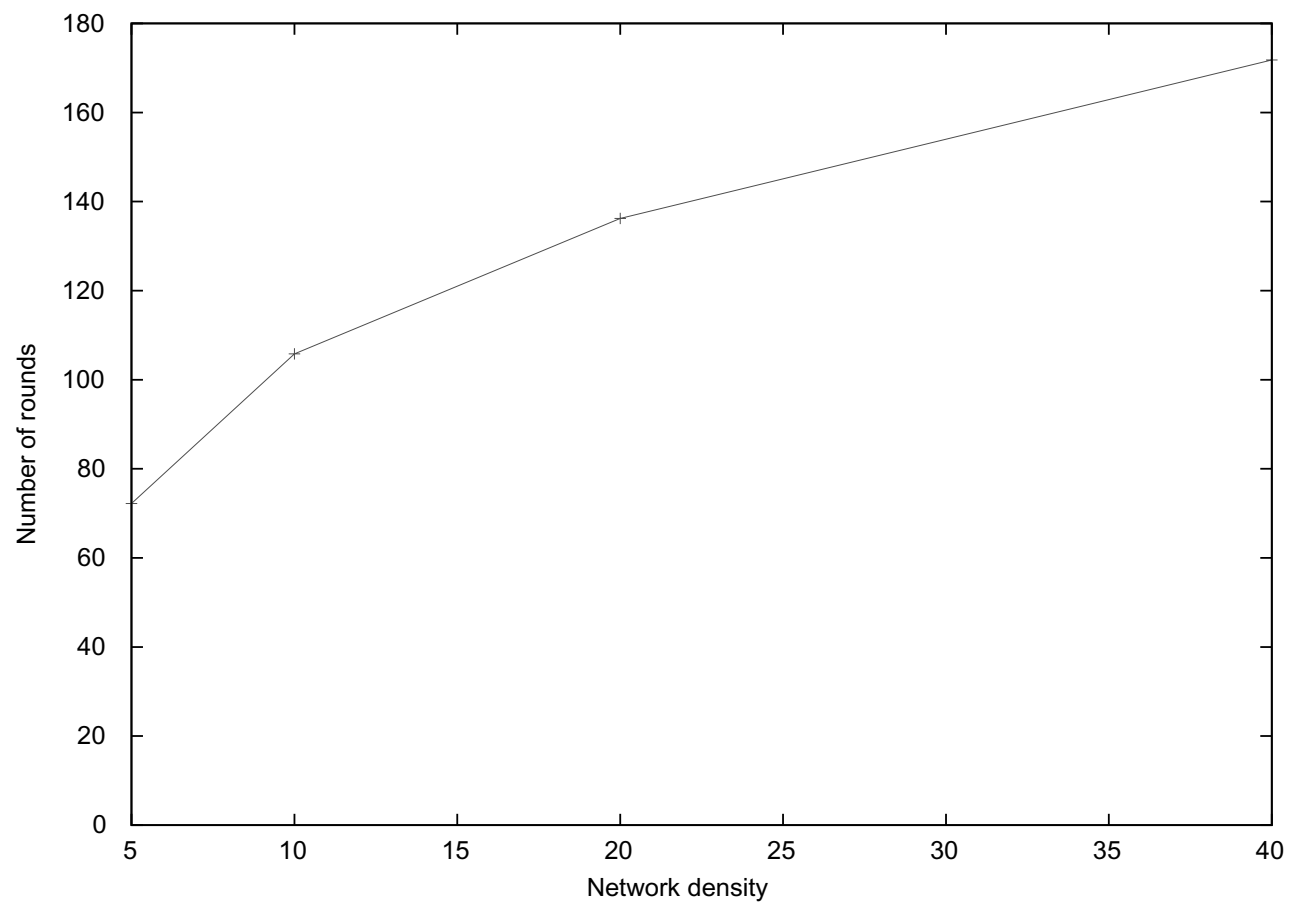

Fig. 6. Number of rounds as a function of network density. 

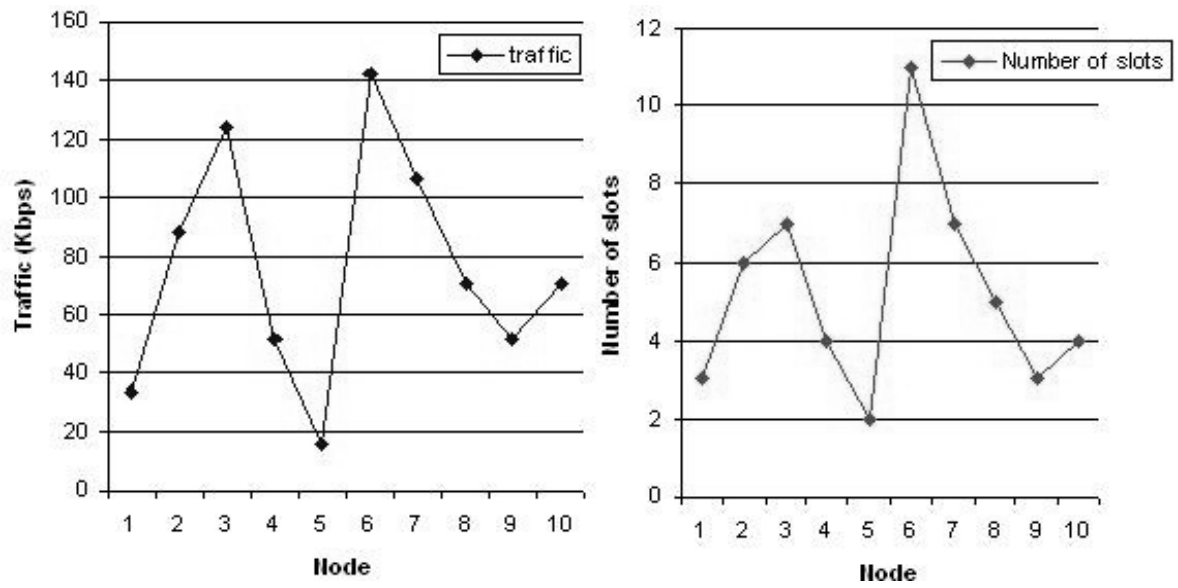

Fig. 7. Slot assignment and traffic rate.

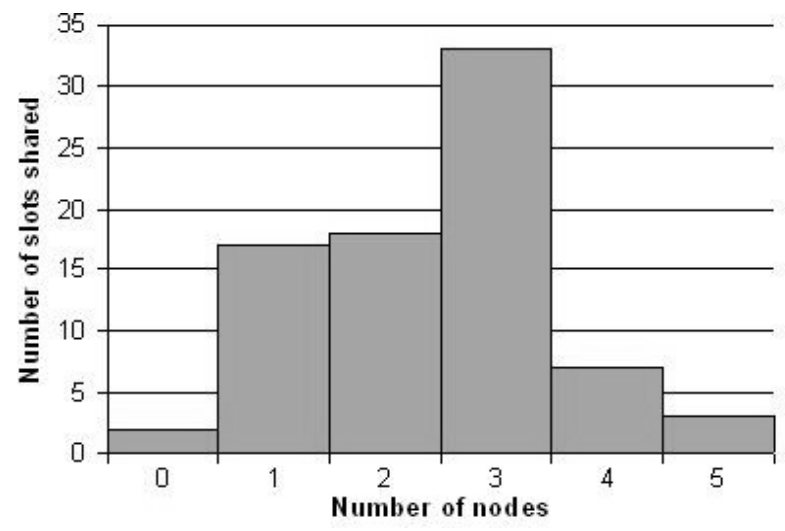

Fig. 8. Slot reuse.

\subsection{Network lifetime and user data delivered}

We now evaluate the performance of SERENA in various wireless ad hoc and sensor networks. More precisely, we quantify the network lifetime obtained with and without SERENA. In both cases, the OLSR routing protocol is used. The simulation parameters are those given in Section 3.2.1. Furthermore, the slot size is fixed to $12 \mathrm{~ms}$ and the frame consists of 80 slots. The frame size is equal to $2 * 4 *$ density, where $4 *$ density is the average number of nodes up to two hops. Simulation results are averaged on 5 simulation runs.

It appears that with SERENA, the network lifetime can be increased by $363 \%$ (see for instance a network of 100 nodes in Fig. 9). Notice that this excellent improvement would be useless, if during this extended network lifetime, the amount of information delivered to the user was not increased. Figure 10 confirms the increase in the delivered data, in about the same proportions.

\subsection{Distribution of node energy consumption}

We now perform the same simulations as those described in Section 3, but now, using SERENA. The new distribution of node energy consumption is illustrated in Fig. 11. The bar diagrams represent the 


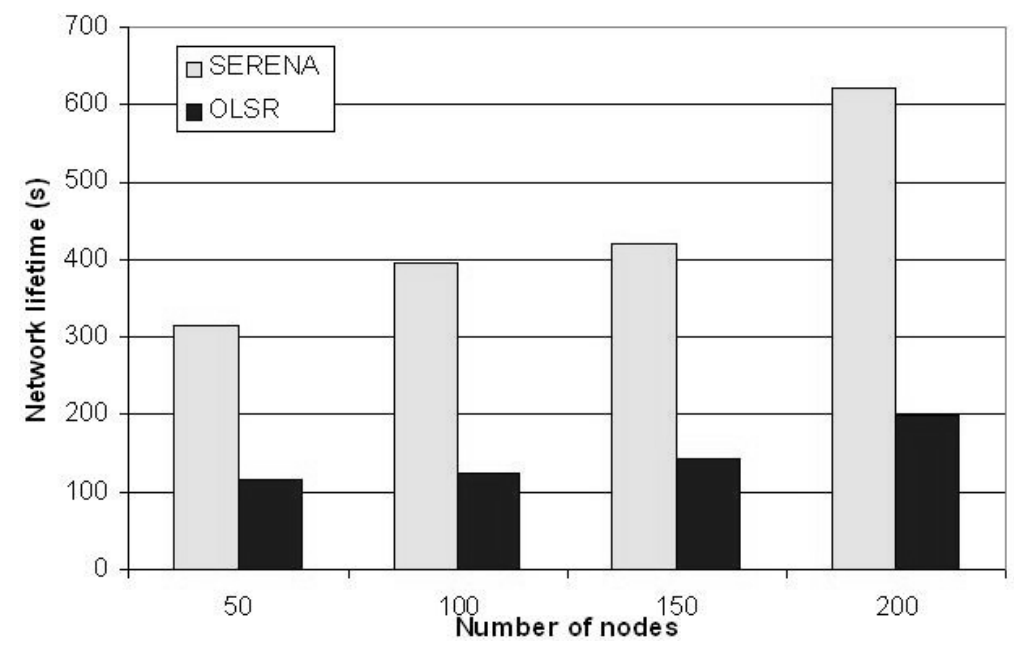

Fig. 9. Network lifetime with and without SERENA.

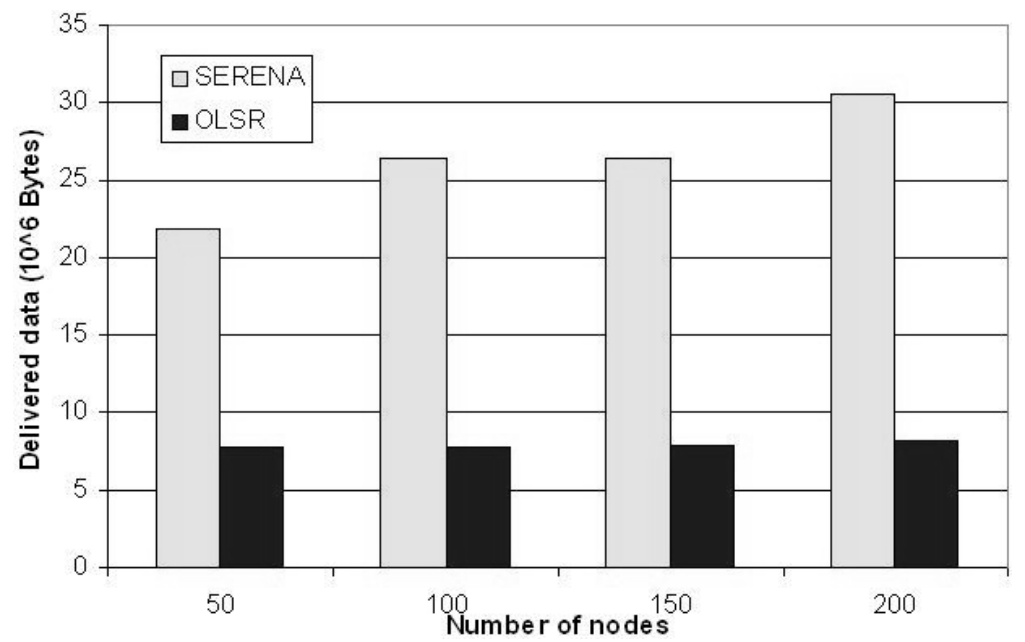

Fig. 10. Amount of user data delivered with and without SERENA.

energy dissipated in the Transmit, Receive, Idle, Overhearing and Interference states successively for different size of networks: 50, 100, 150 and 200 nodes. The network density is set to 10 .

Figure 11 shows that with SERENA, the energy dissipated in the idle state decreases to about $3 \%$ (instead of 50\% as illustrated on Fig. 1). This is the first benefit brought by SERENA. The energy cost due to interferences is about 3\%. It was the second one without SERENA, with about 20\%. This is explained by the fact that with SERENA, if neither the node nor its one-hop neighbors are transmitting, the node is sleeping. Hence, SERENA contributes to significantly reduce the interference phenomenon. This is the second benefit of SERENA. The energy cost due to overhearing can be reduced if messages are sorted according to their destination identifier. 


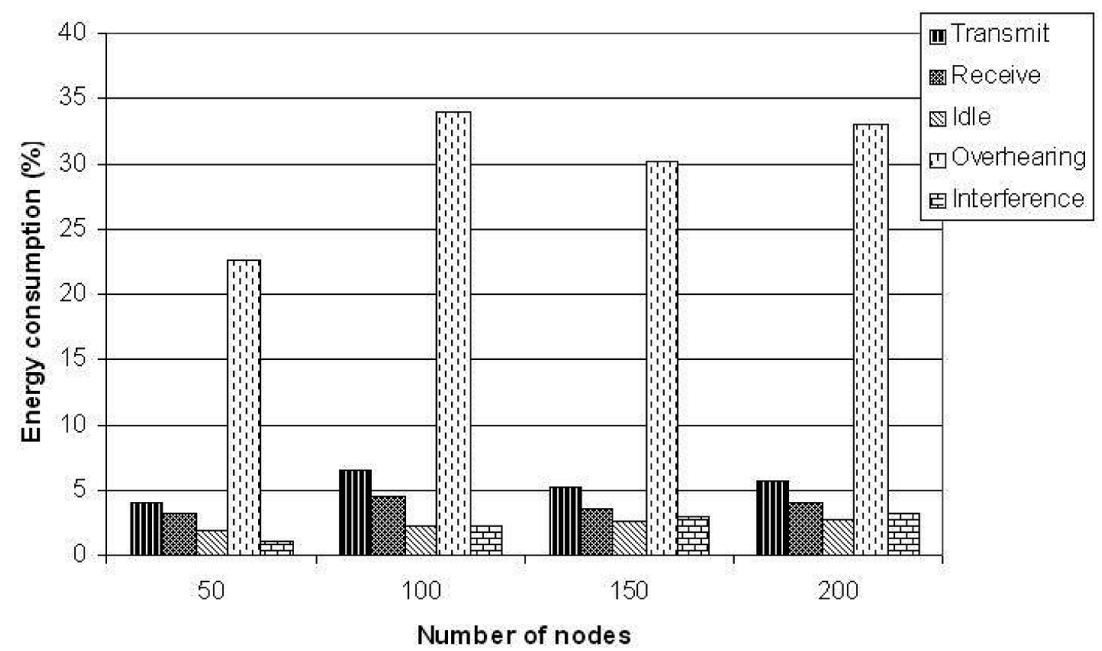

Fig. 11. Distribution of energy consumption with SERENA.

\section{Comparison with TDMA and USAP}

\subsection{TDMA and USAP}

In its basic version, TDMA provides one transmission slot per node in the network. It provides a guaranteed access per cycle for every node and avoids collisions. However, it does not adapt to traffic variations. Many improvements have been proposed in order to take advantage of spatial reuse in wireless networks. Among them, USAP (Unifying Slot Assignment Protocol) $[32,33]$ has drawn a lot of attention.

USAP [32] is a distributed TDMA slot assignment protocol for mobile multihop packet radio networks. It allows any node $N_{i}$ to select a slot for transmission that is unassigned in its neighborhood. A slot is unassigned from $N_{i}$ point's of view if no one-hop neighbor of $N_{i}$ transmits or receives during this slot. In our simulations, we consider an incremental allocation of slots. More precisely, a node requests an additional slot if the number of messages present in its queue is higher than a given threshold.

We will now compare the respective performance of USAP and SERENA in terms of network lifetime, data delivered and node throughput in different wireless ad hoc and sensor networks. These networks are characterized by their number of nodes and density.

\subsection{Simulation parameters}

In the simulations performed in this section, we use the simulation parameters presented in section 3.2.1. Moreover, the size of the slot is set to $12 \mathrm{~ms}$ and the number of slots in a frame is equal to $2 * 4 *$ density. With this slot size, a node can transmit up to four user messages per slot. As these messages can have different MAC destinations, every slot is considered as a broadcast one. The OLSR routing protocol is used with SERENA and USAP. The control overhead induced by SERENA and USAP is not taken into account.

\subsection{Network lifetime and data delivered}

We focus on wireless networks of 100 nodes with density 10 (the average number of neighbors per node). The flow throughput ranges from $8 \mathrm{kbps}$ to $40 \mathrm{kbps}$. We will compare the network lifetime and the amount of data delivered obtained by USAP and SERENA respectively. 


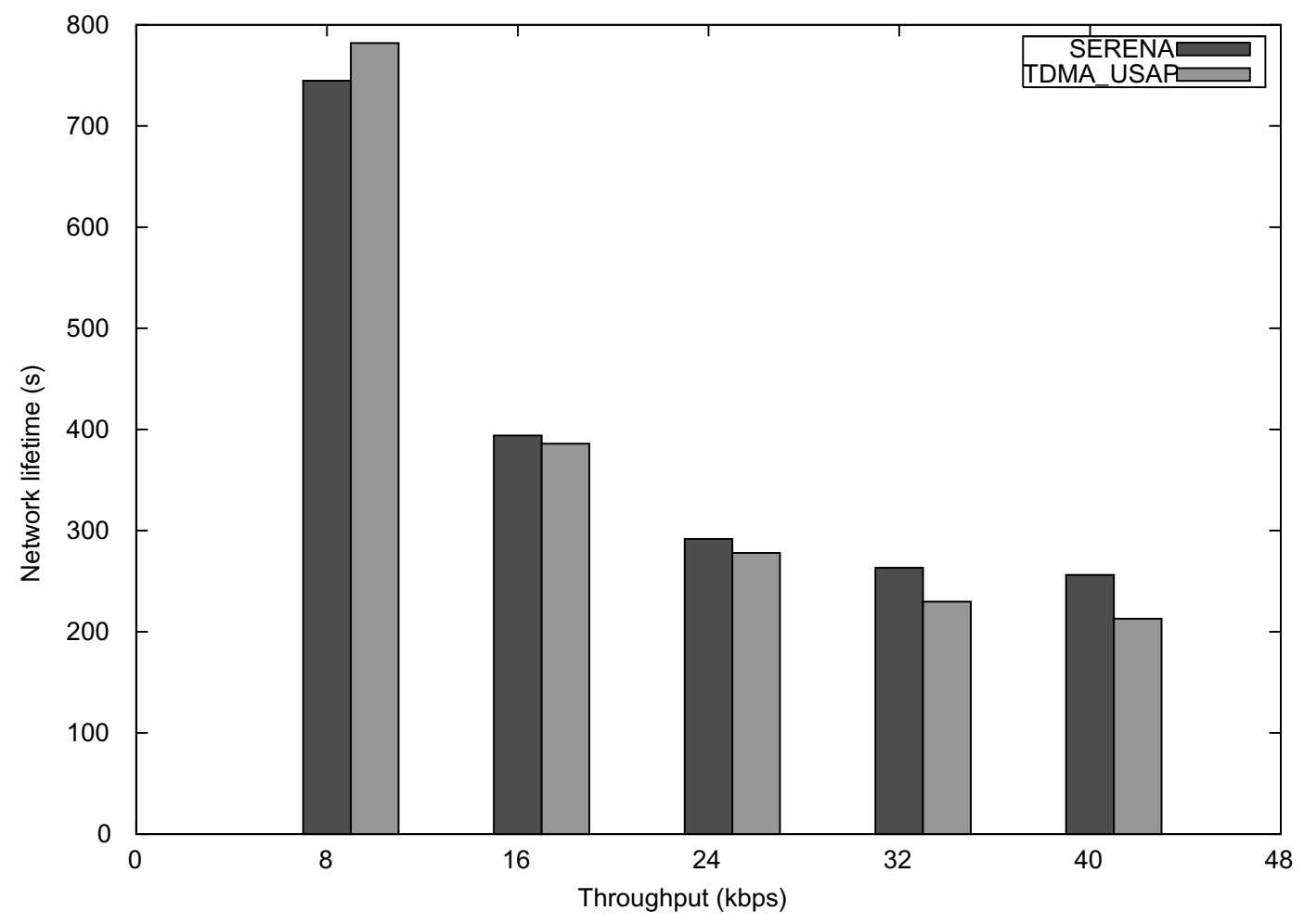

Fig. 12. Network lifetime with SERENA and USAP.

Figures 12 and 13 depict the network lifetime and the amount of data delivered respectively, when the flow throughput is increased. We notice that USAP outperforms SERENA when the throughput is equal to 8kbps. Indeed, in a weakly loaded network, it is sufficient to assign one slot to each node to transmit its traffic. That is why USAP is better than SERENA, which allocates more slots to nodes. However, when the throughput increases, we observe that SERENA becomes more efficient than USAP. We can explain that by the fact that in SERENA, assignment slots is based on:

- colors of nodes which provides an efficient spatial reuse,

- node traffic which allows to allocate the number of slots required to transmit its traffic.

\subsection{Node throughput}

The throughput of a node can be evaluated as follows:

$$
\text { Throughput }(N)=\frac{\text { Number of slots allocated to } N}{\text { Total number of slots }} \cdot \text { Capacity of the medium. }
$$

With classical TDMA, we have

$$
\text { Throughput }_{\mathrm{TDMA}}(N)=\frac{\text { Capacity of the medium }}{\text { Number of nodes }} \text {. }
$$

With SERENA, the maximum throughput of a node $N$ is given by

$$
\text { Throughput }_{\text {SERENA }}(N)=\frac{k^{\prime}+1}{\text { Size }} \cdot \text { Capacity of the medium, }
$$




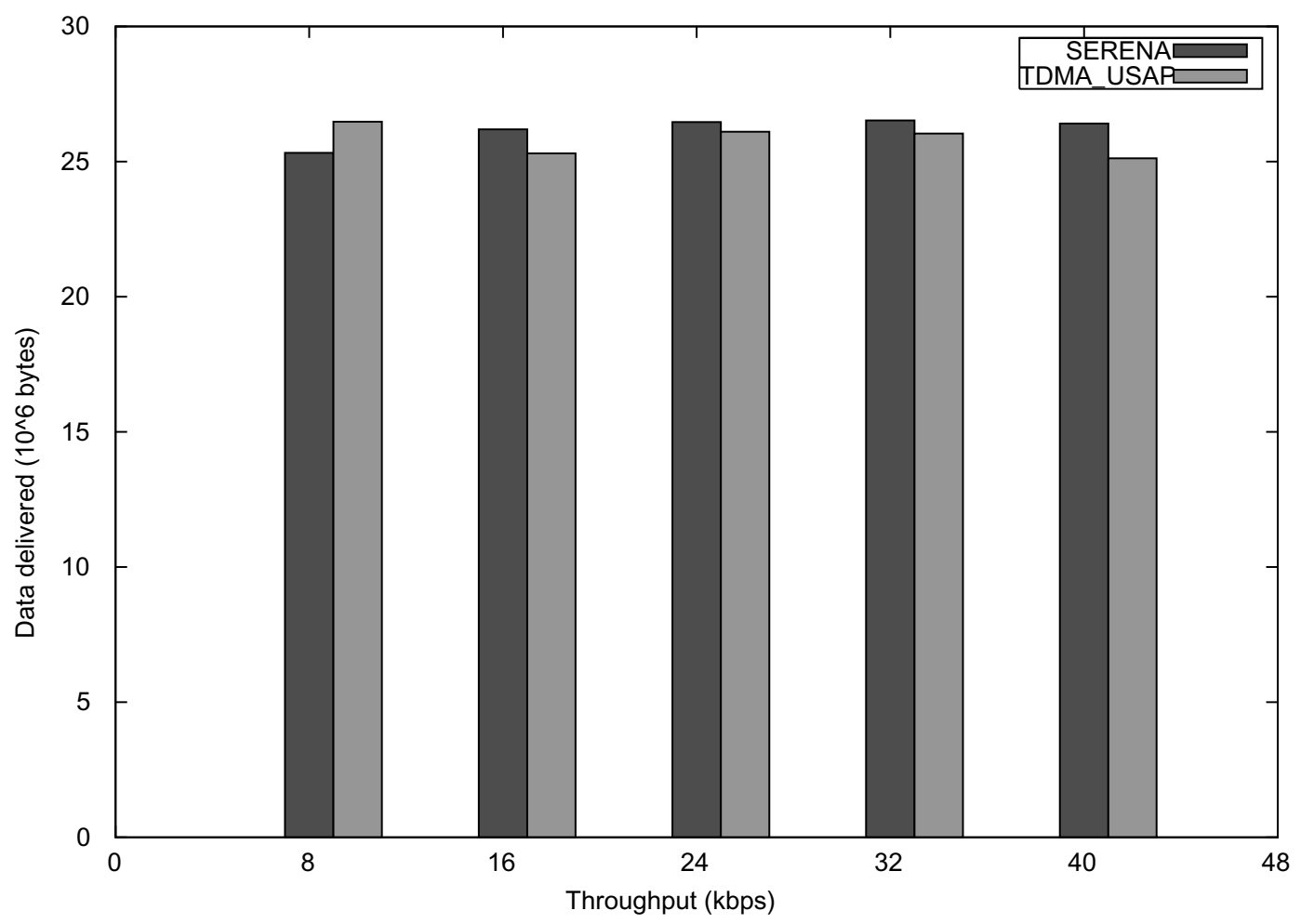

Fig. 13. Amount of user data delivered with SERENA and USAP.

where Size denotes the frame size and $k^{\prime}$ is computed as presented in Section 4.1.

With USAP, the maximum throughput of a node $N$ is given by

$$
\text { Throughput }_{U S A P}(N)=\frac{k^{\prime \prime}+1}{\text { Size }} \cdot \text { Capacity of the medium, }
$$

where Size denotes the frame size and $k^{\prime \prime}$ is determined by the queue size at the end of the slots allocated to the node considered, as presented in Section 6.1. Initially set to $0, k^{\prime \prime}$ is incremented each time an additional slot can be granted to the considered node, provided that neither a 1-hop nor a 2-hop neighbor has the same slot.

Therefore these results show that both SERENA and USAP outperform classical TDMA because of their better spatial reuse. Moreover, SERENA better adapts to changes in traffic requirements. Indeed, the slot assignment of SERENA takes advantage of the colors visible by a node (i.e. colors granted to the one-hop or two-hop neighbors) and shares the slots according to the sum on the number of visible colors, of the maximum slot number granted to each visible color. This is illustrated on Fig. 14.

To compute the slot assignment of node $N$, as represented on Fig. 14, the maximum number of slots used by the two nodes $N_{1}$ and $N_{2}$, up to two-hop from $N$ and colored with the same color, is taken into account by SERENA (i.e. 3 slots). USAP takes the sum (i.e. 5 slots) if $N_{1}$ and $N_{2}$ have taken different slots.

Assuming that all nodes have always a message to send in the network, we evaluate the maximum throughput that can be achieved in a network without routing. The medium capacity is set to $2 \mathrm{Mbps}$. 
Table 2

Maximum throughput in Kbps obtained by a node

\begin{tabular}{lrrrr} 
Nodes & 50 & 100 & 150 & 200 \\
TDMA & 40 & 20 & 13.33 & 10 \\
USAP & 50 & 50 & 50 & 50 \\
SERENA & 125 & 100 & 100 & 75 \\
\hline
\end{tabular}

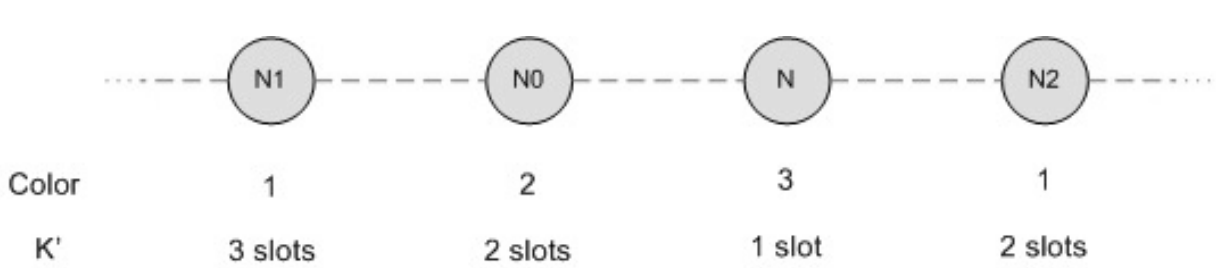

Fig. 14. Slot assignment.

The frame Size, expressed in number of slots, is equal to the number of nodes in classical TDMA and to $2 * 4 *$ density in USAP and SERENA. Each node is assumed to have $4 *$ density neighbors (including one-hop and two-hop neighbors). With USAP and the considered assumptions, a node has one slot each $4 *$ density slots. Hence, with the frame Size considered, it has two slots with USAP. For SERENA, we assume the worst case, where a node sees all the colors. We obtain the results given in Table 2. The throughput of classical TDMA significantly decreases with the number of nodes, whereas the throughput of USAP is kept constant. In all tested networks, the throughput of SERENA is largely better.

We now consider a network of 100 nodes and density 10. Figure 15 compares the throughput obtained by nodes with SERENA and USAP respectively. For clarity, we represent the difference between the granted throughput and the requested throughput.

We observe a better adaption of SERENA to traffic requirements. Hence, all nodes have at least their requested throughput. However, with USAP, some nodes have received less than the requested throughput. These nodes are represented by negative values in Fig. 15.

\section{Conclusion and perspectives}

In this paper, our aim was to maximize network lifetime in wireless ad hoc and sensor networks. We have highlighted that in these networks, most of the node energy is dissipated in the idle state. The second energy consumption comes from interferences. Moreover, as the power consumed by a wireless node in sleeping mode is much smaller than the power consumed in any other mode, the best way to increase network lifetime is to allow nodes to sleep, while ensuring network functionality. Hence, SERENA, in allowing a node to sleep while neither it nor one of its one-hop neighbor is transmitting, contributes considerably to improve energy efficiency. Firstly, the energy dissipated in the idle state is reduced up to $40 \%$. Secondly, the energy loss due to interference becomes negligible (about 3\%). In addition, the node spends more of its time in the Transmit and Receive states: the only useful states from the application point-of-view.

We have compared the performances of SERENA with USAP. Results show that SERENA is more efficient if there is a high load in the network. Since SERENA slot assignment algorithm takes into account the node traffic rate and colors, it provides a good spatial reuse.

We are now studying how to integrate SERENA with an energy efficient routing protocol based on OLSR, avoiding nodes with low residual energy and selecting the route minimizing the energy dissipated. 


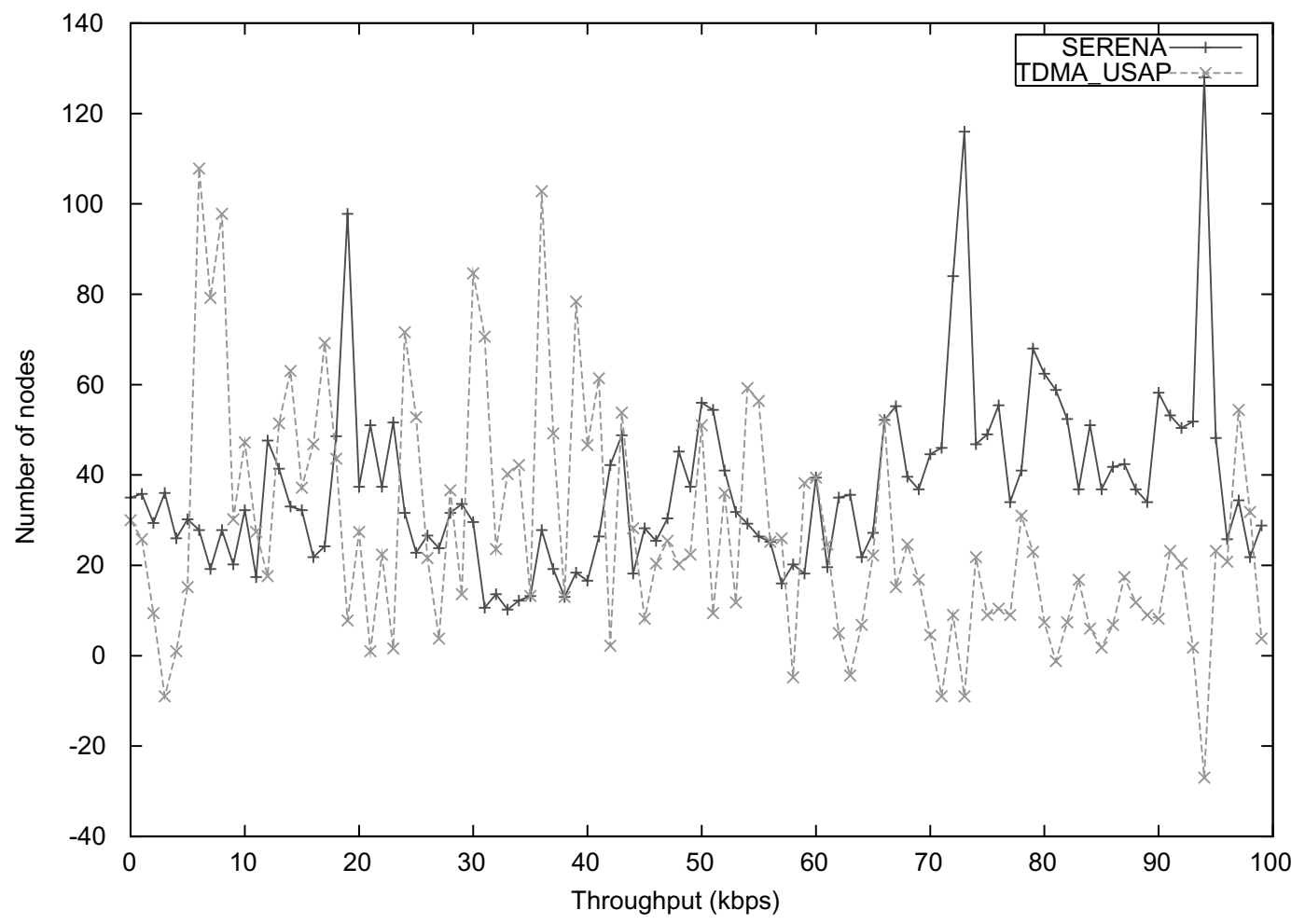

Fig. 15. Node throughput obtained with SERENA and USAP.

\section{Acknowledgment}

This study has been partly funded by the ANR OCARI project.

\section{References}

[1] L. Feeney and M. Nilson, Investigating the Energy Consumption of a Wireless Network Interface in an Ad Hoc Networking Environment, IEEE INFOCOM 2001, Anchorage, Alaska, April 2001.

[2] C. Adjih, T. Clausen, P. Jacquet, A. Laouiti, P. Minet, P. Muhlethaler, A. Qayyum and L. Viennot, Optimized Link State Routing Protocol, RFC 3626, IETF, 2003.

[3] G. Allard, P. Minet, D.Q. Nguyen and N. Shresta, Evaluation of the Energy Consumption in MANET Adhoc-Now 2006, Ottawa, Canada, August 2006.

[4] S. Mahfoudh and P. Minet, An Energy Efficient Routing Based on Olsr in Wireless Ad Hoc and Sensor Networks, PAEWN 2008, Ginowan, Japan, March 2008.

[5] IEEE 802.15, IEEE 802.15.4: Wireless Medium Access Control (MAC) and Physical layer (PHY) specifications for Low-Rate Wireless Personal Area Networks (LR-WPANs), IEEE Computer Society, LAN/MAN standards committee, October 2003.

[6] F. Ingelrest, D. Simplot-Ryl and I. Stojmenovic, Optimal Transmission Radius for Energy Efficient Broadcasting Protocols in Ad Hoc Networks, IEEE Transactions on Parallel and Distributed Systems, June 2006.

[7] M. Cardei, J. Wu and S. Yang, Topology Control in Ad hoc Wireless Networks with Hitch-hiking, First IEEE International Conference on Sensor and Ad hoc Communications and Networks (SECON04), October 2004.

[8] W. Heinzelman, A. Chandrakasan and H. Balakrishnan, Energy-Efficient Communication Protocol for Wireless Microsensor Networks, (Vol. 2), the 33rd Annual Hawaii International Conference on System Sciences (HICSS '00), Maui, Hawaii, USA, pp. 3005-3014, January 2000. 
[9] D. Xia and N. Vlajic, Near-Optimal Node Clustering in Wireless Sensor Networks for Environment Monitoring, AINA 2007, Niagara Falls, Ontario, Canada, May 2007.

[10] K. Kalpakis, K. Dasgupta and P. Namjoshi, Maximum Lifetime Data Gathering and Aggregation in Wireless Sensor Networks, University of Maryland Baltimore County (UMBC), August 2002.

[11] X. Tang and J. Xu, Extending Network Lifetime for Precision-Constrained Data Aggregation in Wireless Sensor Networks, IEEE INFOCOM 2006, Barcelona, Spain, April 2006.

[12] J. Kulik, W. Rabiner and H. Balakrishnan, Adaptive Protocols for Information Dissemination in Wireless Sensor Networks, 5th ACM/IEEE Mobicom Conference, Seattle, WA, August 1999.

[13] D.Q. Nguyen and P. Minet, Scalabilty of the OLSR protocol with the Fish Eye extension, ICN 2007, Sainte Luce, Martinique, April 2007.

[14] R.C. Shah and J.M. Rabaey, Energy aware routing for low energy ad hoc sensor networks, IEEE Wireless Communications and Networking conference (WCNC) 1 (March 2002), 17-21.

[15] D. Ganesan, R. Govindan, S. Shenker and D. Estrin, Highly-Resilient, energy-efficient multipath routing in wireless sensor networks, CM SIGMOBILE Mobile Computing and Communications Review (MC2R) 1(2) (2001).

[16] A. Srinivas and E. Modiano, Minimum Energy Disjoint Path Routing in Wireless Ad-Hoc Networks, MOBICOM'2003, September 2003.

[17] S.-M. Senouci and G. Pujolle, Energy Efficient Routing In Wireless Ad Hoc Networks, (Volume 7), IEEE International Conference on Communications (ICC 2004), pp. 4057-4061, June 2004.

[18] S. Kwon and Ness B. Shroff, Energy-Efficient Interference-Based Routing for Multi-hop Wireless Networks, IEEE INFOCOM'2006, Barcelona, Spain, April 2006.

[19] H. Hassanein and Jing Luo, Reliable Energy Aware Routing In Wireless Sensor Networks, Second IEEE Workshop on Dependability and Security in Sensor Networks and Systems DSSNS, April 2006.

[20] N.Shresta, Reception Awarness for Energy Conservation in Ad Hoc Networks, PhD Thesis, Macquarie University Sydney, Australia, November 2006.

[21] M. Cardei, M. Thai, Y. Li and W. Wu, Energy-Efficient Target Coverage in Wireless Sensor Networks, IEEE INFOCOM 2005, Miami, Florida, March 2005.

[22] J. Carle and D. Simplot-Ryl, Energy-efficient area monitoring for sensor networks, Computer 37(2) (Febuary, 2004), 40-46.

[23] M. Cardei and D. Du, Improving Wireless Sensor Network Lifetime Through Power Aware Organization, ACM Journal of Wireless Networks, May 2005.

[24] D. Brelaz, New methods to color the vertices of a graph, Communications of the ACM 22(4), 1979.

[25] J. Peemoller, A correction to Brelaz's modification of Brown's coloring algorithm, Communications of the ACM 26(8), 1983.

[26] J. Hansen, M. Kubale, L. Kuszner and A. Nadolski, Distributed Largest-First Algorithm For Graph Coloring, EURO-PAR 2004, Pisa, Italy, August 2004.

[27] T. Herman and S. Tixeuil, A Distributed TDMA Slot Assiognment Algorithm for Wireless Sensor Networks, ALGOSENSORS 2004, Turku, Finland, July 2004.

[28] L. Bao and J.J. Garcia-Luna-Aceves, A New Approach to Channel Access Scheduling for Ad Hoc Networks, MOBICOM 2001, Rome, Italy, July 2001.

[29] C. Busch, M. Magdon-Ismail, F. Sivrikaya and B. Yener, Contention-Free Mac Protocols for Wireless Sensor Networks, DISC 2004, Amsterdam, Netherlands, October 2004.

[30] V. Rajendran, K. Obraczka and J.J. Garcia-Luna-Aceves, Energy-Efficient, Collision-Free Medium Access Control for Wireless Sensor Networks, Sensys'03, Los Angeles, California November 2003.

[31] V. Rajendran, J.J. Garcia-Luna-Aceves and K. Obraczka, Energy-efficient, application-aware medium access for sensor networks, IEEE MASS 2005, Washington, November 2005.

[32] C.D. Young, USAP: a Unifying Dynamic Distributed Multichannel TDMA Slot Assignment Protocol, (Vol. 1), IEEE MILCOM 1996, October 1996.

[33] C.D. Young, USAP Multiple Access: Dynamic Resource Allocationfor Mobile Multihop Multichannel Wireless Networking, (Vol. 1), IEEE MILCOM 1999, November 1999.

[34] P. Jacquet, Analytical results on connected dominating sets in mobile ad hoc networks INRIA Research Report, RR 5173, http://www.inria.fr/rrrt/rr-5173, April 2004.

[35] A. Nasipuri and S. Das, On-Demand Multipath Routing for Mobile Ad Hoc Networks, Int. Conf. on Computer Communications and Networks, Boston, MA, October 1999.

Pascale Minet is Vice Head of the HIPERCOM (High Performance Communication) project-team at INRIA (French National Research Institute for Research in Computer Science and Control). Her main research areas include wireless ad hoc and sensor networks, energy efficiency, node activity scheduling, energy efficient routing, multicast in MANETs, quality of service 
and real-time scheduling. She is co-author of the OLSR routing protocol and many book chapters. She is member of the program committee of international conferences such as HPCC 2008, ICCCN2008, IFIP Wireless Days 2008, Med-Hoc-Net 2008, PAEWN2008, SERA 2008, SNPD 2008, SPECTS 2008. She is co-chair with Giorgio Buttazzo of the RTNS 2008 conference program committee. Previously, she was member of the editorial board of two international journals: IJCS and IJCIS. In 1998, she qualified from the University of Versailles (France) in advising PhD students. Her web page can be accessed http://hipercom.inria.fr/rminet/.

Saoucène Mahfoudh received her Master degree in Network and Computer Science from the University of Paris 6 in 2006. She is currently preparing her PhD thesis at INRIA in the HIPERCOM project-team. She is interested in energy efficient routing, graph coloring, node activity scheduling and cross layer design in wireless ad hoc and sensor networks. 

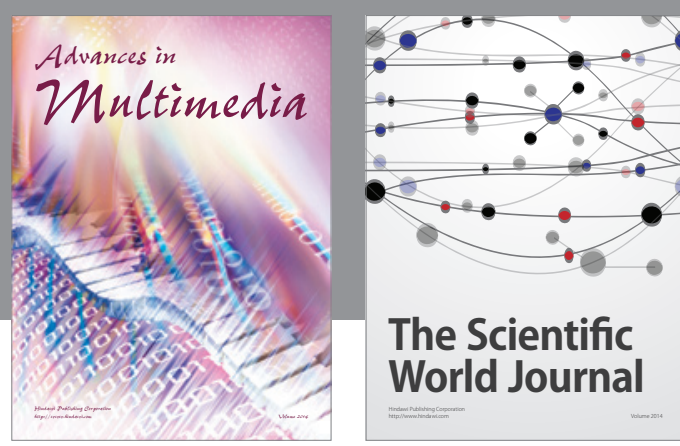

The Scientific World Journal
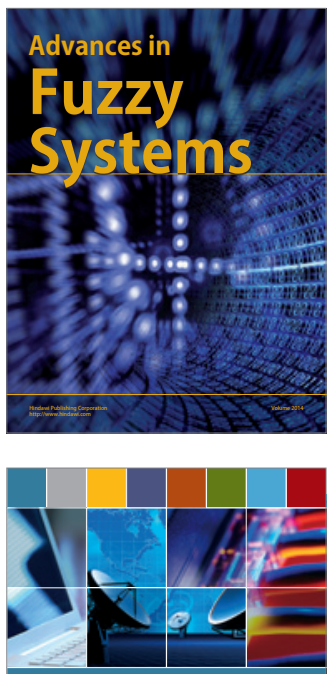

Computer Networks and Communications
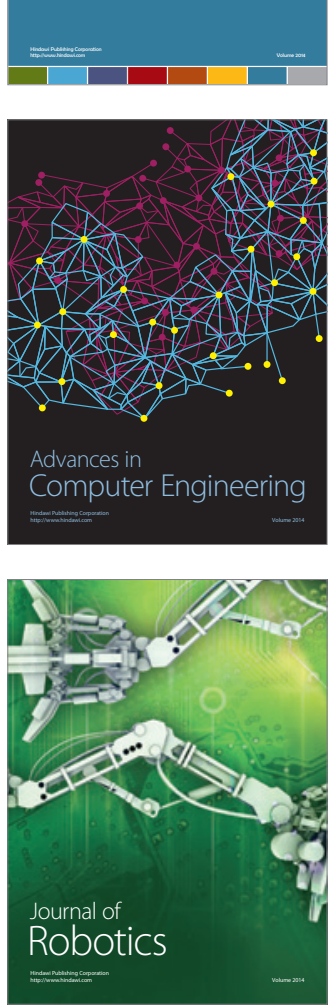
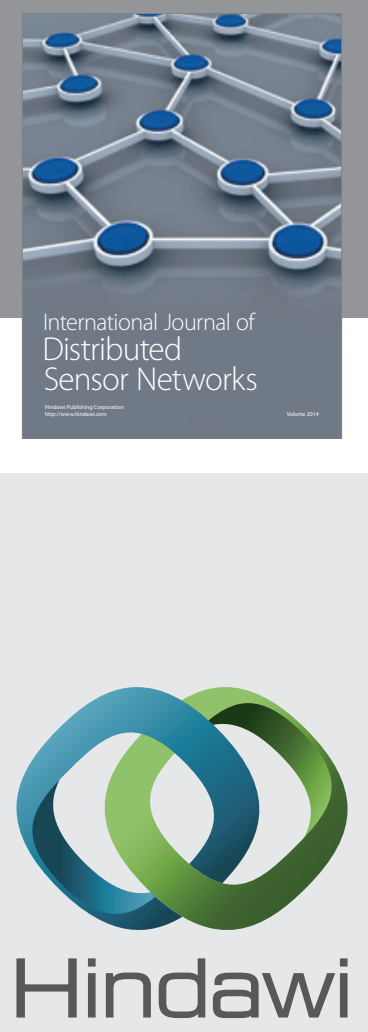

Submit your manuscripts at

http://www.hindawi.com
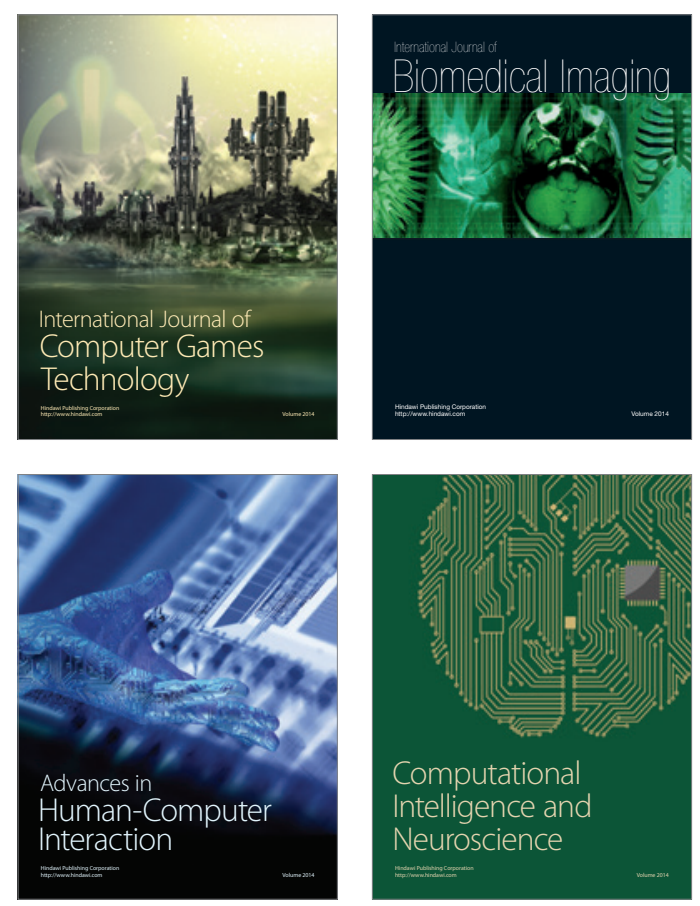
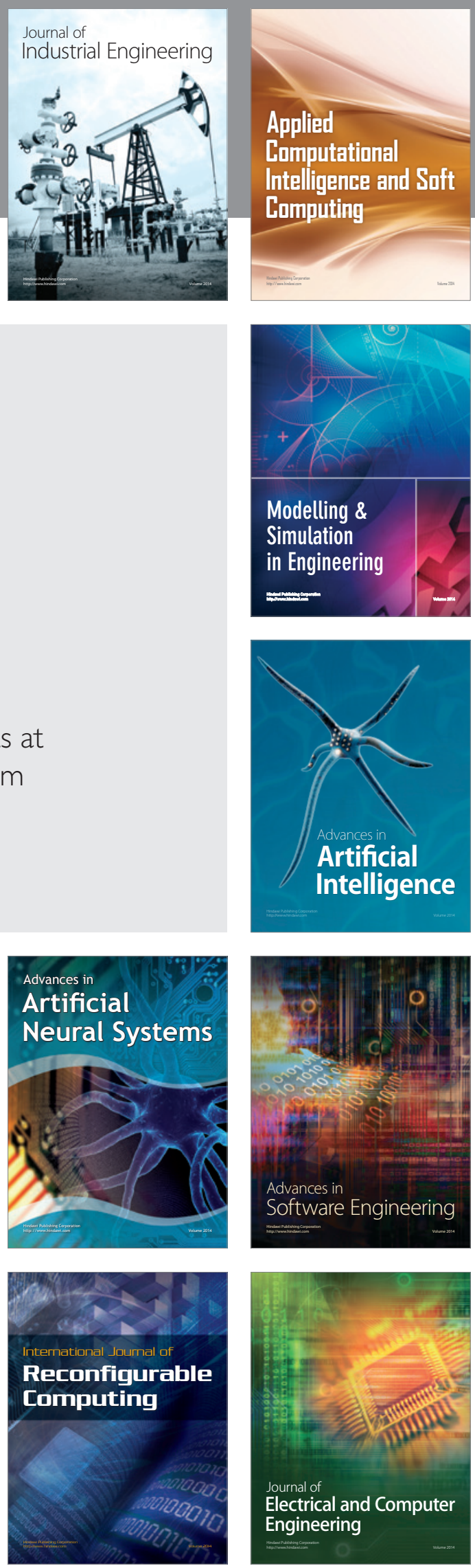